\title{
Hybrid Distributed Acoustic-Temperature Sensing Using a Few-Mode Fiber
}

\author{
Islam Ashry, ${ }^{\mathrm{a}, \dagger}$ Yuan Mao, ${ }^{\mathrm{a}, \dagger}$ Tien Khee Ng, ${ }^{\mathrm{a}}$ Frode Hveding, ${ }^{\mathrm{b}, \#}$ Muhammad Arsalan, ${ }^{\mathrm{b}}$ \\ and Boon S. Ooi ${ }^{\mathrm{a},{ }^{*}}$ \\ ${ }^{a}$ King Abdullah University of Science and Technology (KAUST), Computer, Electrical, and \\ Mathematical Sciences and Engineering, Thuwal 23955-6900, Saudi Arabia; ${ }^{b}$ EXPEC Advanced \\ Research Center, Saudi Aramco, Dhahran 34464, Saudi Arabia \\ ${ }^{\dagger}$ These authors contributed equally to this work \\ "\#frode.hveding@aramco.com, *boon.ooi@kaust.edu.sa
}

\begin{abstract}
Optical fiber distributed acoustic sensor (DAS) and distributed temperature sensor (DTS) are considerably desirable for many important applications including oil and gas industry. Simultaneous measurements of vibration and temperature will exclude the need for two separate DAS and DTS systems, reduce overall cost, and ensure continuous real-time monitoring of these two important sensing parameters. We here devise a hybrid DAS-DTS system using a few-mode fiber (FMF). Although the system requirements for DAS and DTS are quite different, FMF is considered an ideal compromise to satisfy the requirements of the two systems.
\end{abstract}

Keywords: Acoustic sensors, temperature sensor, optical fiber sensors, optical fibers, optical sensors.

\section{INTRODUCTION}

Fiber optic distributed sensors have been utilized in many important applications including oil and gas industry [1], realtime monitoring of structural health [2], aerospace transportation [3], among others. In the oil and gas industry, for instance, numerous sensing parameters are recorded within the life of an oil/gas well, starting from the drilling process and throughout the producing period. Once the production begins, monitoring vibrations and temperatures of the downhole fluid is significantly beneficial to optimize the production process [1]. This can be achieved through using optical fiber distributed acoustic sensing (DAS) [4] and distributed temperature sensing (DTS) [5]. The underlying operation principle of DAS system relies on detecting backscattered Rayleigh signals from an optical fiber, while optical pulses from a narrow linewidth laser source are injected into the fiber. On the other hand, DTS extracts temperatures profile along an optical fiber via comparing the fiber Stokes and anti-Stokes Raman backscattered signals when launching high-power optical pulses into the fiber.

Generally, monitoring of vibrations and temperatures distributions are achieved by using two different fiber optic sensing systems. Designing a hybrid DAS-DTS system will exclude the need for two separate sensors, reduce overall cost, and ensure continuous real-time monitoring of vibrations and temperatures. However, combining the two systems is challenging because their implementations require different operational conditions. In particular, the backscattered power of the Raman signal within an optical fiber is roughly 30-40 dB less than that of the Rayleigh signal. Consequently, for DTS systems, the Stokes and anti-Stokes signals need to be averaged to improve the signal to noise ratio (SNR) of a measured temperature profile. Moreover, optical pulses of a high pump power should be employed which would induce nonlinearity in the standard single mode fiber (SMF) [6]. This fiber nonlinearity, such as stimulated Raman scattering, inhibits the proper operation of DTS systems. Alternatively, multimode fibers (MMFs), which have higher nonlinearity threshold in comparison with SMFs, are used for DTS systems. However, it is difficult to offer MMF-based DAS system because MMF supports propagating a large number of modes and each mode encounters independent interference behavior which turns out to producing a Rayleigh signal independent on vibrations.

We here tackle this challenge: Designing a hybrid DAS-DTS system using a few-mode fiber (FMF). FMFs have been deployed in many sensing applications including designing a large scale absorption-based sensing network [7], reconstructing spatial distributions of gases that diffuse into a FMF [8], monitoring multiple parameters simultaneously 
[9], etc. To resolve the fiber nonlinearity dilemma and simultaneously keep the DAS operation reliable, we use the FMF as a compromise between the SMF and MMF [10]. The threshold power of nonlinearity of a typical FMF is higher than that of the SMF. Consequently, compared with the SMF case, optical pulses of a higher pump power can be injected into the FMF which would improve the SNR of the DTS system. On the other hand, the FMF guides a restricted number of spatial modes such that one can easily control and manage intermodal coupling and differential mode group delays within it, which make the FMF promising for the DAS system. In particular, we excite only the fundamental mode into the FMF such that the FMF operates in a quasi-single-mode (QSM) state. Meanwhile, in the backward direction, we collect the Rayleigh signals associated with the fundamental mode only. This strategy significantly reduces the noise produced by intermodal coupling in corresponding MMF-based DAS. The work presented here would find interests from many industrial applications.

\section{SYSTEM DESIGN OF THE FMF-BASED DAS}

We start with showing the possibility of using a FMF for DAS and in the following section we proceed to the design of the FMF-based hybrid DAS-DTS system. The experimental setup of the reported DAS system designed using a FMF is shown schematically in Figure 1. A narrow linewidth laser source generates a continuous wave (CW) light of a $1555 \mathrm{~nm}$ wavelength and a $40 \mathrm{~mW}$ optical power. The $\mathrm{CW}$ light is then modulated into optical pulses using an acousto-optic modulator (AOM) which is driven by a function generator. Each pulse has a $100 \mathrm{~ns}$ width and the modulation repetition rate of the pulses is $10 \mathrm{kHz}$. The power of the optical pulses is amplified using an erbium-doped fiber amplifier (EDFA1) and then the pulses are injected through a SMF-based circulator into a $\sim 3.1 \mathrm{~km}$ FMF.

The used FMF supports propagating the $\mathrm{LP}_{01}, \mathrm{LP}_{11 \mathrm{a}}$, and $\mathrm{LP}_{11 \mathrm{~b}}$ modes at the selected operation wavelength. In the forward pump direction, we excite only the fundamental mode $\left(\mathrm{LP}_{01}\right)$ into the FMF such that the FMF operates in a QSM state. This can be achieved via aligning the axes of the SMF and FMF, followed by splicing them or even joining them with a mating-sleeve [11]. Near the end of the FMF, we wind a $10 \mathrm{~m}$ section of it around a piezoelectric transducer (PZT) cylinder such that the vibration amplitude and frequency of the PZT cylinder can be well-controlled using a function generator [12]. As the fundamental mode propagates along the FMF, Rayleigh signal is backscattered as a combination of the entire modes supported by the FMF [13]. Receiving the Rayleigh signal as a mixed of spatial modes would create significant noise for the DAS system. Fortunately, the circulator's SMF acts as a spatial mode filter in the
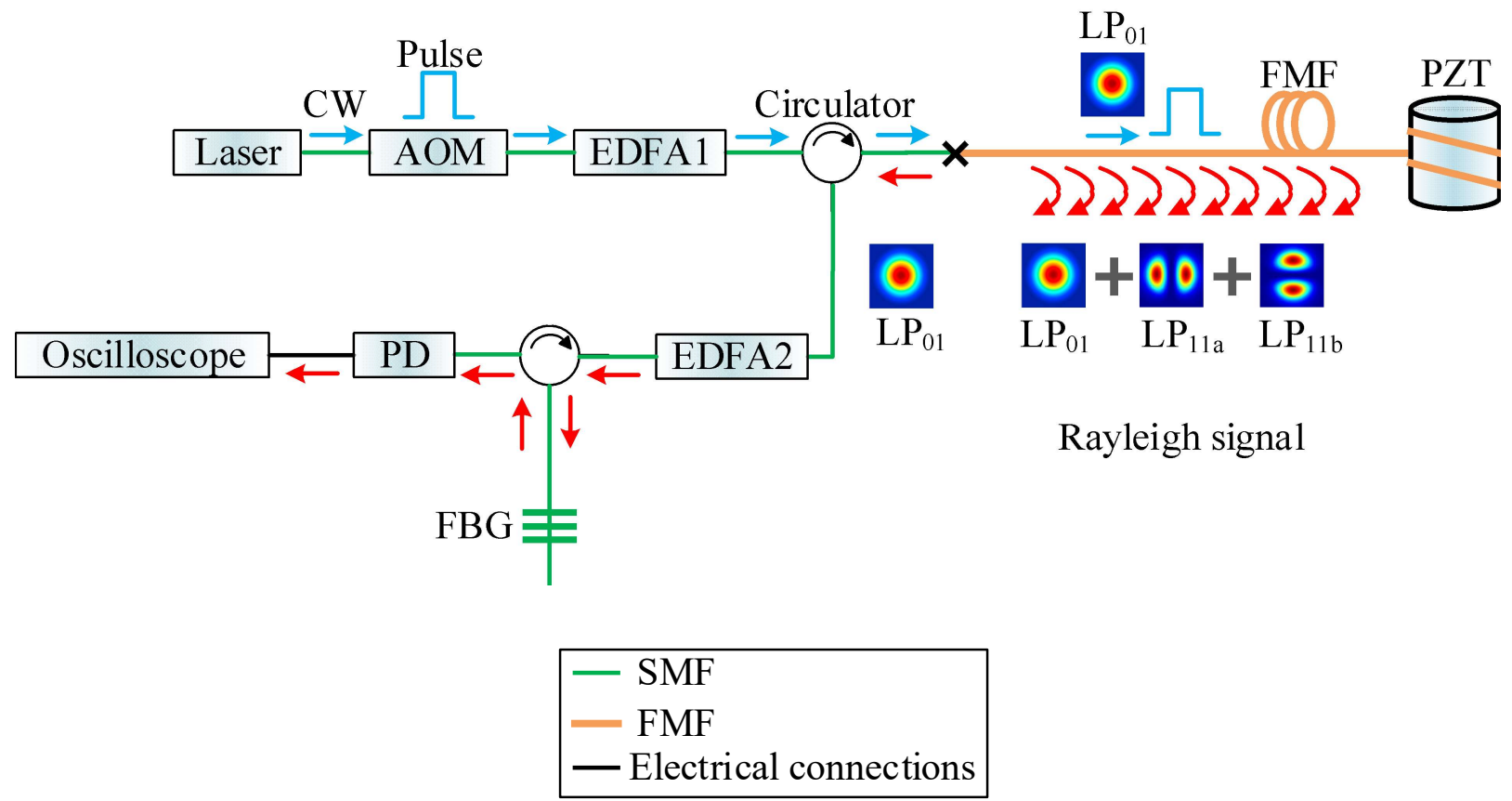

Figure 1. Experimental setup of the DAS system using a FMF. 
backward direction such that only the $\mathrm{LP}_{01}$ mode can go through the SMF and the other higher order modes are filtered out. The filtered back-reflected Rayleigh signal is amplified with another erbium-doped fiber amplifier (EDFA2) which amplified spontaneous emission (ASE) noise is discarded with a fiber Bragg grating (FBG). Eventually, the Rayleigh signal is detected with a photodetector (PD) and sampled at a $200 \mathrm{MS} / \mathrm{s}$ rate using an oscilloscope.

In order to validate the QSM-operation of the FMF, we use a CCD camera to capture the intensity profile of the output light from the FMF. Figures 2(a) and 2(b) show two temporal representative examples when using the center-launch technique to excite the fundamental mode into the FMF. Clearly, roughly a pure $\mathrm{LP}_{01}$ mode is excited and propagate along the FMF. On the other hand, when exciting a higher order mode $\left(\mathrm{LP}_{11 \mathrm{a}}\right)$ at the input end of the FMF, the intensity profiles at the FMF's output end are unstable because of the easily mode coupling between the degenerate $\mathrm{LP}_{11 \mathrm{a}}$ and $\mathrm{LP}_{11 \mathrm{~b}}$ modes [Figure 2(c) and 2(d)].
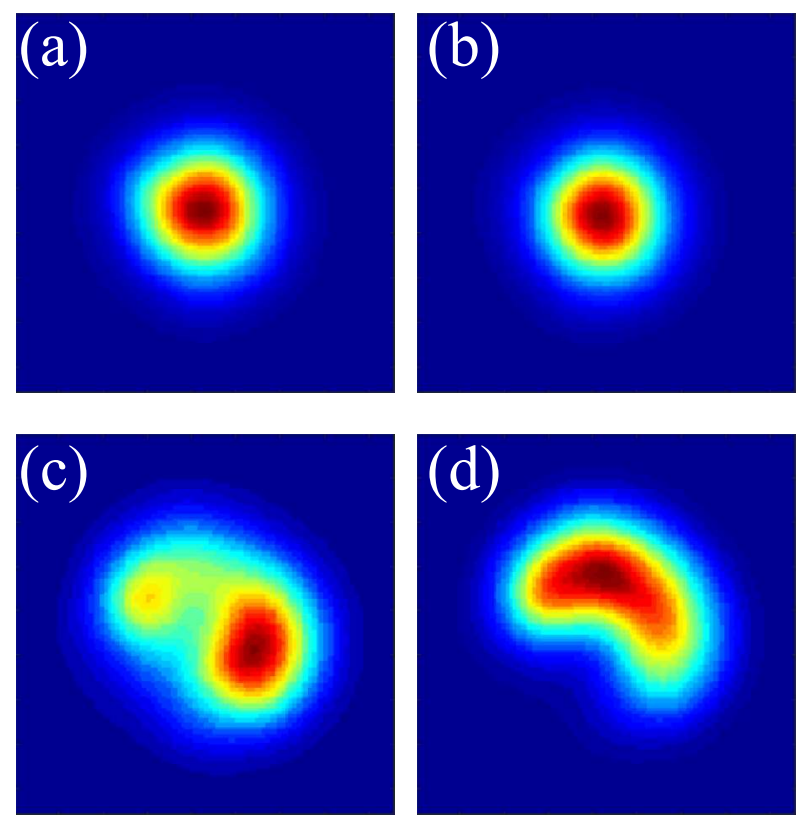

Figure 2. Temporal changes of the intensity profiles at the FMF output port when injecting the $\mathrm{LP}_{01}(\mathrm{a}-\mathrm{b})$ and $\mathrm{LP}_{11 \mathrm{a}}$ mode (c-d) into the FMF.

When launching an $l m$ mode into a FMF, the power of backscattered Rayleigh signal received as $l$ ' $m$ ' mode at the FMF's input port is approximated as [14]:

$$
P_{i m^{\prime}}^{l m}(t)=P_{o} \gamma_{s} \Gamma_{i^{\prime} m}^{l m}, \bar{v} \Delta T e^{-2 \alpha \bar{v} t},
$$

where $t$ is the time, $P_{o}$ and $\Delta T$ represent the power and width of the injected pulses into the FMF, respectively, and $\gamma_{s}$ denotes the ratio between the total backscattered Rayleigh power and the incident power. $\bar{v}=\left(v_{g}^{l m} v_{g}^{i m^{\prime}}\right) /\left(v_{g}^{l m}+v_{g}^{i m^{\prime}}\right) ; v_{g}^{l m}$ and $v_{g}^{i m{ }^{\prime}}$ are the group velocities of the $l m$ and $l^{\prime} m$ ' mode, respectively, and $\alpha$ denotes the optical attenuation coefficient of the FMF. The parameter $\Gamma_{i m^{\prime}}^{m m}$ is defined as the ratio of the backscattered Rayleigh power as $l^{\prime} m$ ' mode to that total backscattered Rayleigh power [14]:

$$
\Gamma_{i m}^{l m} \propto \int_{0}^{2 \pi} \int_{0}^{\infty} \varphi_{l m}^{2}(r, \phi) \varphi_{i^{\prime \prime}}^{2}(r, \phi) r d r d \phi
$$


where $\varphi_{l m}(r, \phi)$ and $\varphi_{i^{\prime}{ }^{\prime}}(r, \phi)$ respectively represent the spatial near field distribution of the $l m$ and $l^{\prime} m$ mode. Following Eqs. (1) and (2), when injecting an $l m$ mode into the FMF, the backscattered Rayleigh signal is dominant by the same $l m$ mode. This is because the parameter $\Gamma_{i^{\prime} m^{\prime}}^{l m}$ is maximized when $l^{\prime} m^{\prime}=l m$. These two equations also validate our design of the FMF-based DAS because we launch only the fundamental mode into the FMF to receive a Rayleigh signal that is dominant by the same mode. Afterwards, we pick up only the fundamental mode and filter out the other higher order modes to mitigate the noise in the FMF-based DAS.

In our experiment, as aforementioned, the PZT cylinder is located near the end of the FMF and we set its vibration frequency to $200 \mathrm{~Hz}$. Figure 3(a) shows a representative example of 10 consecutive Rayleigh traces recorded when using the QSM-operated FMF. Applying the differential algorithm, subtracting the temporal Rayleigh traces from the initial recorded one [4], we can identify the location of vibration along the FMF [Figure 3(b)]. The vibration frequency of the PZT cylinder can also be calculated by applying the fast Fourier transform (FFT) to the differential signal at the PZT location. Figure 3(c) shows the power spectrum of the PZT vibration event. The results of Figures 3(b) and 3(c) clearly indicate the success of using the FMF for DAS system.
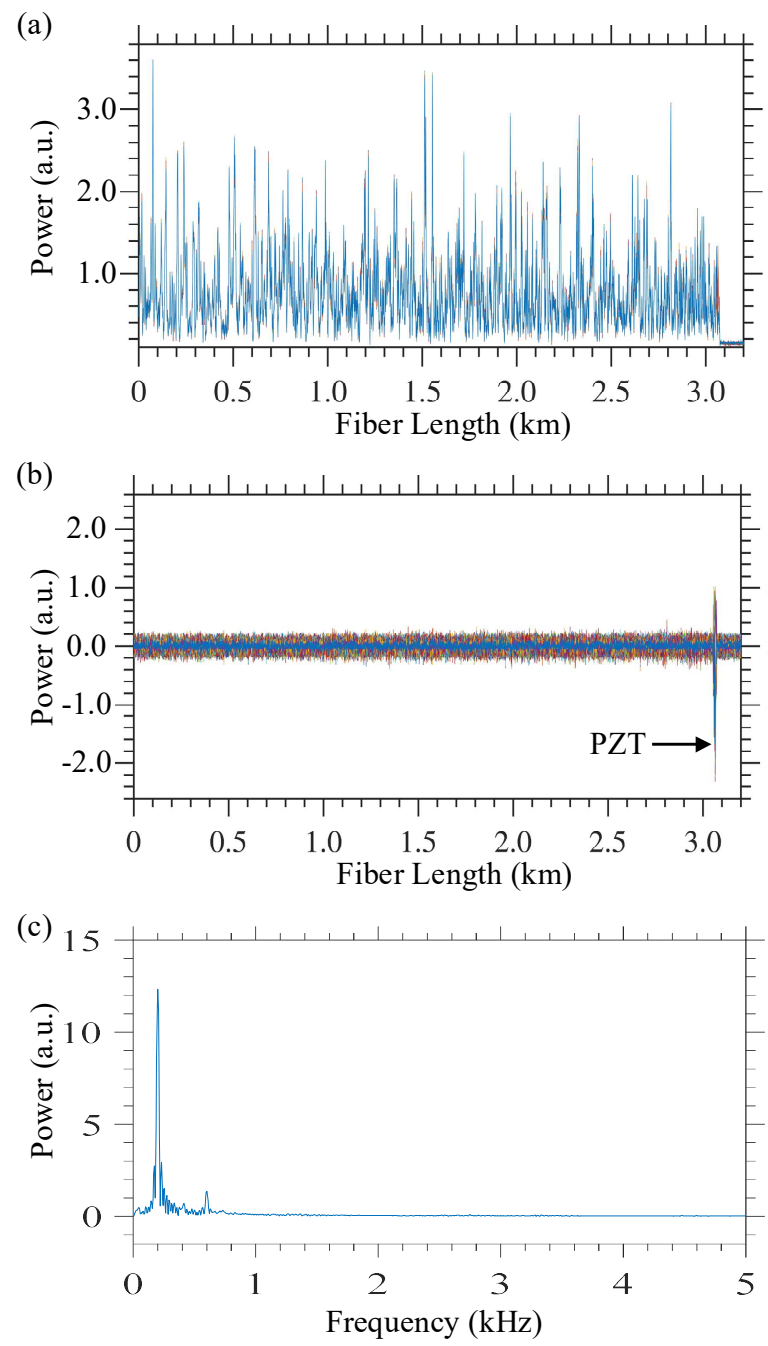

Figure 3. (a) Consecutive Rayleigh traces (a) and differential signal (b) recorded when using the QSM-operated FMF. (c) Power spectrum of the $200 \mathrm{~Hz}$ PZT vibration event. 


\section{HYBRID DAS-DTS USING THE FMF}

We here modify the former experimental setup such that we can use the reported system to monitor vibration and temperature simultaneously. The FMF-based hybrid DAS-DTS system is shown schematically in Figure 4 where the narrow linewidth laser generates a CW light to be modulated into optical pulses (100ns width, $10 \mathrm{kHz}$ repetition rate) using the AOM. The optical signals are amplified with EDFA1 to be launched through a SMF-based circulator into a multimode fiber (MMF) of $50 \mu \mathrm{m} / 125 \mu \mathrm{m}$ core/cladding diameter. We use MMF between the SMF and FMF because it is difficult to find a commercially available FMF-based wavelength division multiplexing (WDM) filter. As a proof-ofconcept demonstration, we use a MMF-based WDM filter instead. Again, we use the conventional center-launching method between the SMF and MMF to roughly only excite the fundamental mode into the MMF.

The bidirectional WDM filter can spectrally resolve Rayleigh, Stokes Raman, and Anti-Stokes Raman signals. Since Rayleigh scattering occurs at the same wavelength of the input pump light, the optical pulses get into the WDM filter through its Rayleigh port [Figure 4]. Again the center-launching method is applied between the MMF and FMF at the output port of the WDM filter to mainly excite the fundamental mode in the FMF. While an optical pulse is propagating along the FMF, Rayleigh and Raman signal of the whole modes are backscattered. In the backward direction, the bidirectional WDM filter separates the Rayleigh, Stokes Raman, and Anti-Stokes Raman signal. The two Raman signals are directed towards an Avalanche photodiode (APD) and oscilloscope for light detection and data sampling, respectively. Using the Stokes and Anti-Stokes Raman signals, we can extract the temperature distribution along the FMF [15]. On the other hand, the spectrally filtered backscattered Rayleigh signal is forwarded towards the SMF for the purpose of vibration sensing, as we demonstrated in Section 2.
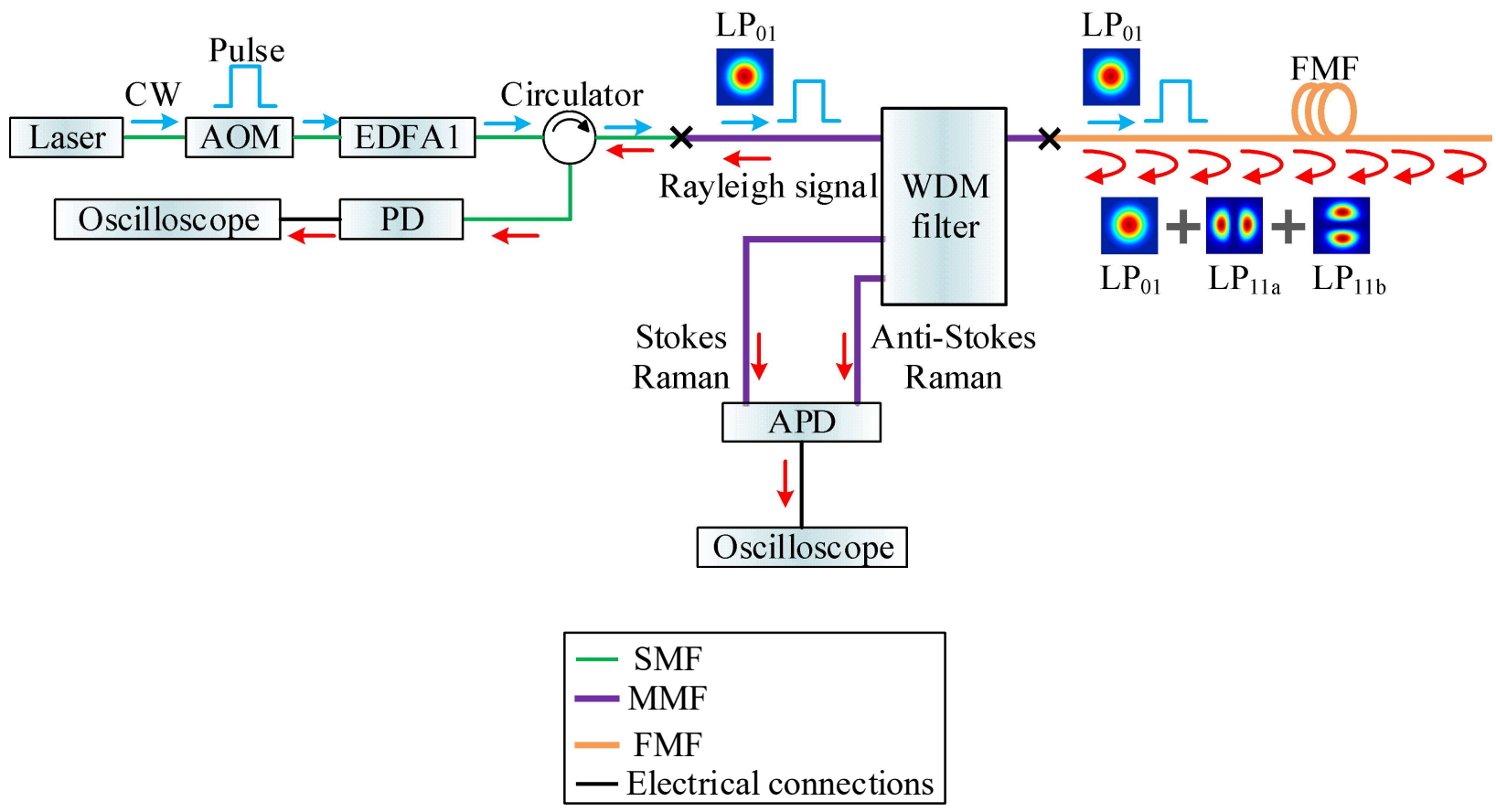

Figure 4. Experimental setup of the hybrid DAS-DTS system using a FMF.

As a proof-of-concept demonstration, we place a $\sim 1.9 \mathrm{~km}$ section of the FMF at room temperature and carry out the temperature sensing experiment. Figure 5(a) and Figure 5(b) show representative examples of the recorded Anti-Stoke and Stokes signals. By linking the ratio between the anti-Stokes and Stokes signals with the temperature [15], we can calculate the temperature distribution along the FMF [Figure 5 (c)]. These results show the possibility of using the FMF for temperature sensing. 

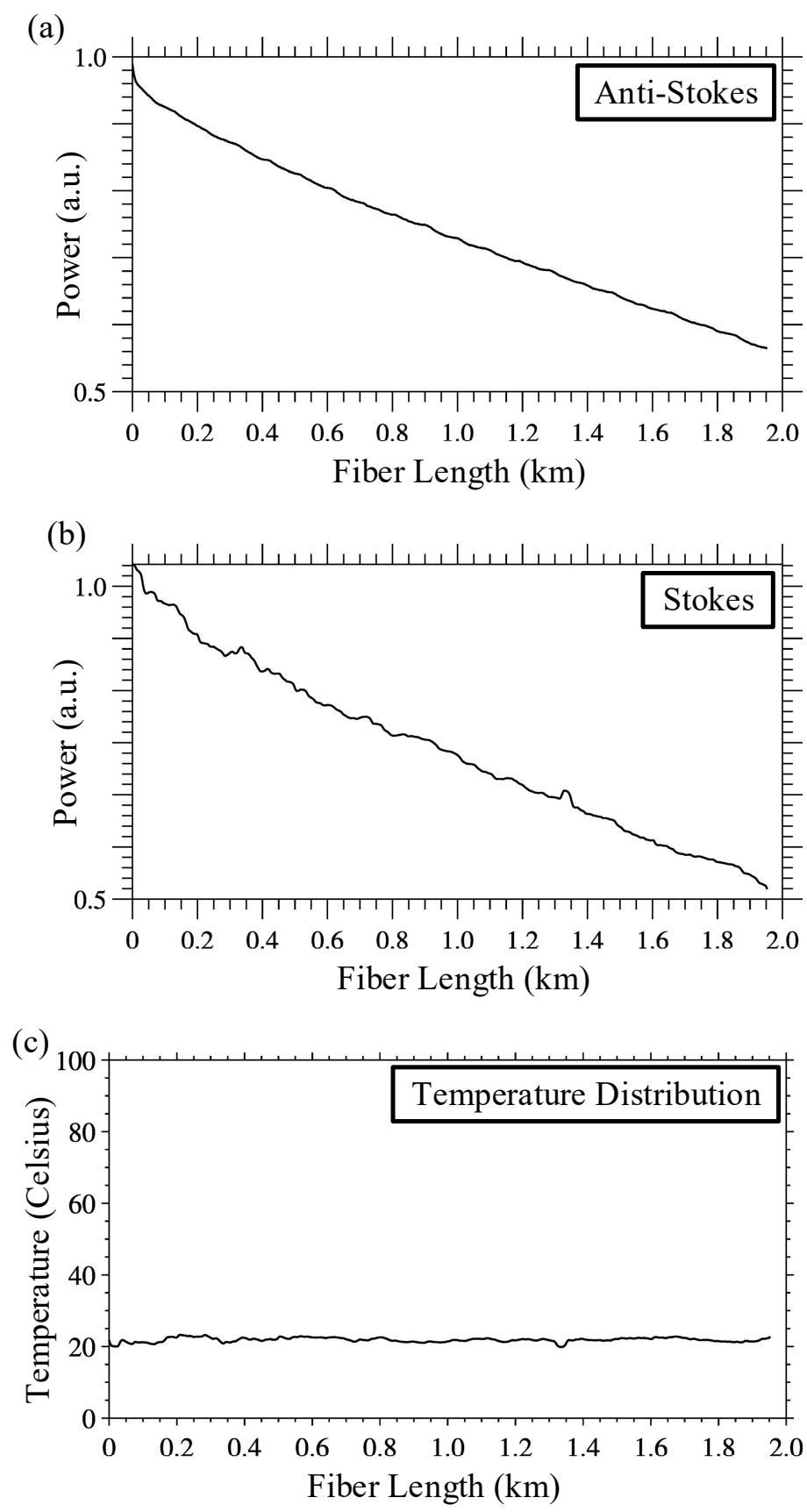

Figure 5. Representative examples of the Anti-Stokes (a) and Stokes (b) Raman signals recorded using the FMF. (c) Temperature distribution along the FMF when it is placed at room temperature.

\section{CONCLUSION}

In summary, we use a FMF as a compromise between the standard SMF and MMF to design a hybrid DAS-DTS system. By using the center-launching technique, we excite only the fundamental mode into the FMF to satisfy the operation requirements of the DAS and DTS. Vibrations produced by a PZT cylinder and a temperature distribution of about $22{ }^{\circ} \mathrm{C}$ are well-measured using the reported system. This work would find many important applications including oil and gas industry. 


\section{ACKNOWLEDGEMENTS}

The authors gratefully acknowledge the financial support provided to this work by the Saudi Aramco under Grant No. RGC/3/3138-01. This work is partially supported by King Abdullah University of Science and Technology (KAUST) baseline funding (BAS/1/1614-01-01), KAUST equipment funding KCR/1/2081-01-01, and GEN/1/6607-01-01.

\section{REFERENCES}

[1] Yamate, T., Fujisawa, G. and Ikegami, T., "Optical Sensors for the Exploration of Oil and Gas," J. Light. Technol. 35(16), 3538-3545 (2017).

[2] Barrias, A., Casas, J. R. and Villalba, S., "A review of distributed optical fiber sensors for civil engineering applications," Sensors (Switzerland) 16(5), 748 (2016).

[3] Merlo, S., Malcovati, P., Norgia, M., Pesatori, A., Svelto, C., Pniov, A., Zhirnov, A., Nesterov, E. and Karassik, V., "Runways ground monitoring system by phase-sensitive optical-fiber OTDR," 4th IEEE Int. Work. Metrol. AeroSpace, Metroaerosp. 2017 - Proc., 523-529 (2017).

[4] Bao, X., Zhou, D. P., Baker, C. and Chen, L., "Recent Development in the Distributed Fiber Optic Acoustic and Ultrasonic Detection,” J. Light. Technol. 35(16), 3256-3267 (2017).

[5] Bao, X. and Chen, L., "Recent Progress in Distributed Fiber Optic Sensors," Sensors (Switzerland) 12(7), 86018639 (2012).

[6] Agrawal, G., [Nonlinear Fiber Optics] (2013).

[7] Ashry, I., Wang, A. and Xu, Y., "Mode-division-multiplexing of absorption-based fiber optical sensors," Opt. Express 24(5), 5186 (2016).

[8] Ashry, I., Wang, A. and Xu, Y., "Mode-Based Reconstruction of Chemical Distributions in Optical Fibers," IEEE J. Sel. Top. Quantum Electron. 23(2) (2017).

[9] Wu, H., Tang, M., Wang, M., Zhao, C., Zhao, Z., Wang, R., Liao, R., Fu, S., Yang, C., Tong, W., Shum, P. P. and Liu, D., "Few-mode optical fiber based simultaneously distributed curvature and temperature sensing," Opt. Express 25(11), 12722 (2017).

[10] Wang, M., Wu, H., Tang, M., Zhao, Z., Dang, Y., Zhao, C., Liao, R., Chen, W., Fu, S., Yang, C., Tong, W., Shum, P. P. and Liu, D., "Few-mode fiber based Raman distributed temperature sensing," Opt. Express 25(5), 4907 (2017).

[11] Mao, Y., Ashry, I., Alias, M. S., Ng, T. K., Hveding, F., Arsalan, M. and Ooi, B. S., "Investigating the Performance of a Few-Mode Fiber for Distributed Acoustic Sensing," IEEE Photonics J. 11(5), 1-10 (2019).

[12] Ashry, I., Mao, Y., Alias, M. S., Ng, T. K., Hveding, F., Arsalan, M. and Ooi, B. S., "Normalized differential method for improving the signal-to-noise ratio of a distributed acoustic sensor," Appl. Opt. 58(18), 4933 (2019).

[13] Wang, Z., Wu, H., Hu, X., Zhao, N., Mo, Q. and Li, G., "Rayleigh scattering in few-mode optical fibers," Sci. Rep. 6 (2016).

[14] Wang, Z., Wu, H., Hu, X., Zhao, N., Mo, Q. and Li, G., "Rayleigh scattering in few-mode optical fibers," Sci. Rep. 6 (2016).

[15] Ma, X., Chang, J., Wang, Z., Wang, W. and Li, T., "Using multiple reference points in Raman based distributed temperature sensor for eliminating DC interference,” IEEE Sens. J. 14(1), 295-301 (2014). 